\title{
Degenerative Makulopathien (bei jüngeren Patienten)
}

\section{Degenerative Maculopathy (in Younger Patients)}

\author{
G. Haas, B. Treiblmayr, M. Kralinger, N. E. Bechrakis
}

Universitätsklinik für Augenheilkunde und Optometrie, Medizinische Universität Innsbruck \begin{abstract}
Makulopathien sind Erkrankungen und daraus resultie-
rende Funktionseinschränkungen der Netzhautmitte (Makula). Den fortschreitenden Funktionsverlust durch chronische Veränderung von Gewebe in der Makula bezeichnet man als Makuladegeneration.
\end{abstract}

In diesem Artikel werden folgende Netzhauterkrankungen mit Befall des Netzhautzentrums bei jüngeren Menschen beschrieben:
- zentrale seröse Chorioretinopathie (ZSCR)

- Grubenpapille

- myope Makulopathie

- Aderhautfalten (Striae)

- Angioid Streaks

Davon abzugrenzen ist die altersassoziierte Makuladegeneration (AMD) des älteren Menschen.

\section{Zentrale seröse Chorioretinopathie}

\section{Definition}

Die zentrale seröse Chorioretinopathie (ZSCR) ist die vierthäufigste Retinopathie nach AMD, diabetischer Retinopathie und venösen Gefäßverschlüssen. Die Erstbeschreibung geht auf Albrecht von Graefe im Jahr 1866 zurück. Seither gibt es für diese Erkrankung immer wieder unterschiedliche Bezeichnungen. Der derzeit gebräuchlichste Begriff, zentrale seröse Chorioretinopathie, wurde 1967 von Gass geprägt.

\section{Epidemiologie}

In einer retrospektiven Studie aus Minnesota, USA, in der alle von 1980 - 2002 neu aufgetretenen ZSCR-Fälle analysiert wurden, wurde eine Inzidenz von 9,9/ 100000 bei Männern und 1,7/100000 bei Frauen beschrieben (Kitzmann et al. 2008). Das Durchschnittsalter der Patienten liegt zwischen 45 und 51 Jahren. Ältere Patienten (> 50 Jahre) präsentieren sich häufiger mit RPE-Verlust, choroidalen Neovaskularisationen und bilateraler Beteiligung. Obwohl berichtet wird, dass bei bis zu 40\% der Fälle beide Augen betroffen sind, ist die Rate bei Erstdiagnose mit 4\% wesentlich geringer.

\section{Abkürzungen}

$\begin{array}{ll}\text { A2E } & \text { N-Retinyliden-N-Retinylethanolamin } \\ \text { AMD } & \text { altersassoziierte Makuladegeneration } \\ \text { C.C. } & \text { cum correctione (mit Korrektur) } \\ \text { CNV } & \text { choroidale Neovaskularisation } \\ \text { DOG } & \text { Deutsche Ophthalmologische Gesellschaft } \\ \text { EDI } & \text { enhanced Depth Imaging } \\ \text { FAG } & \text { Fluoresceinangiografie } \\ \text { ICG } & \text { Indocyaninangiografie } \\ \text { mfERG } & \text { multifokales Elektroretinogramm } \\ \text { OCT } & \text { optische Kohärenztomografie } \\ \text { PDT } & \text { photodynamische Therapie } \\ \text { PEA } & \text { Pigmentepithelabhebung } \\ \text { PM } & \text { pathologische Myopie } \\ \text { PXE } & \text { Pseudoxanthoma elasticum } \\ \text { RPE } & \text { retinales Pigmentepithel } \\ \text { SD-OCT } & \text { Spectral Domain optische Kohärenztomografie } \\ \text { TTT } & \text { transpupillare Thermotherapie } \\ \text { VEGF } & \text { vascular endothelial Growth Factor } \\ \text { VPDT } & \text { photodynamische Therapie mit Verteporfin } \\ \text { ZSCR } & \text { zentrale seröse Chorioretinopathie }\end{array}$


Eine erhöhte Prävalenz einer bestimmten ethnischen Gruppe zuzuordnen, ist aufgrund der geringen Datenlage sehr schwierig. Dennoch gibt es Hinweise, dass Asiaten häufiger bilaterale bzw. multifokale Formen aufweisen und Schwarzafrikaner einen schlechteren Endvisus haben im Vergleich zu Kaukasiern.

\section{Risikofaktoren}

Es gibt eine Reihe von Risikofaktoren, die für die Bildung einer ZSCR zumindest mitverantwortlich sind (s. Infobox).

\section{Risikofaktoren}

Risikofaktoren für die Bildung einer zentralen serösen Chorioretinopathie (ZSCR)

- Typ-A-Persönlichkeit

- systemische Steroideinnahme

- Cushing-Syndrom

- Schwangerschaft

- Kollagenosen

- obstruktive Schlafapnoe

- Antibiotikaeinnahme

- Alkohol und Tabak

- allergisch bedingte Atemwegserkrankung

- nicht behandelte arterielle Hypertonie

- Einnahme von Psychopharmaka

- Einnahme von Antihistaminen

- Helicobacter pylori

- Familienanamnese

- Testosteronspiegel

Personen, die psychosozialen Belastungen ausgesetzt sind und weniger Kompensationsmechanismen haben, können häufiger eine ZSCR entwickeln. Auch andere Persönlichkeitsmerkmale wie emotionale Instabilität, Unsicherheit, aber auch Flexibilität und Spontanität, scheinen für die Entwicklung einer ZSCR eine Rolle zu spielen. Dennoch können Personen auch in einer stabilen psychosozialen Lebenssituation von einer ZSCR betroffen sein.

Man erklärt sich das mit einem höheren Spiegel an zirkulierenden Katecholaminen und Kortikosteroiden, wobei die Katecholaminspiegel bei Typ-A-Persönlichkeit $4 \times$ und die Kortikosteroidspiegel $40 \times$ höher in Menschen mit Typ-A-Persönlichkeit verglichen mit der Typ-B-Persönlichkeit sind. Untersuchungen haben ge-

\section{Hintergrund}

Typ-A- versus Typ-B-Persönlichkeit

- Typ A: wettbewerbsorientiert, ungeduldig, oft aggressiv mit der Tendenz, Kontrolle auszuüben

- Typ B: eher geduldig, entspannt, wenig wettbewerbsorientiert, gelassener als Typ-A-Persönlichkeit

zeigt, dass bei Patienten mit ZSCR die Katecholamine Adrenalin und Noradrenalin erhöht sind und die Plasmakonzentration von Adrenalin stark mit der Makuladicke korreliert. Der endogene Cortisolspiegel ist ungefähr um 50\% erhöht. Untersuchungen, dass Betablocker den sympathischen Stress senken und damit die Wahrscheinlichkeit des Wiederauftretens von ZSCR reduzieren, stützen diese These.

Das Cushing-Syndrom wird sowohl endogen induziert, z.B. durch Tumoren, als auch exogen durch Zufuhr von Steroiden in unterschiedlichen Verabreichungsformen hervorgerufen. Viele publizierte ZSCR-Fälle bei systemischer Steroideinnahme werden als bilateral, mit subretinalen Ablagerungen beschrieben und für die Entwicklung einer chronischen ZSCR mit verantwortlich gemacht.

Es werden auch nach Organtransplantationen ZSCRFälle beschrieben, wobei bei 1 - $6 \%$ der Nierentransplantierten ein besonderes Risiko für die Entwicklung einer ZSCR besteht. Hier sollen neben der langen Einnahme von systemischen Steroiden Faktoren, die die choroidale Durchblutung beeinflussen, wie arterielle Hypertension, Mikroangiopathien und Hämodialyse, eine weitere Rolle spielen. Auch nach Knochenmarktransplantation und Graft-versus-Host-Reaktion wurden ZSCR-Fälle beschrieben.

Schwangere haben ebenfalls ein höheres Risiko, eine ZSCR zu entwickeln. Dies kann auch auf den steigenden endogenen Kortikosteroidspiegel zurückzuführen sein. Die ZSCR entsteht meist im 3. Trimenon und bildet sich innerhalb von 1-2 Monaten nach der Geburt zurück. Unklar hingegen ist weiterhin die häufige Assoziation von ZSCR und weißen subretinalen Ablagerungen bei Schwangeren.

\section{Klinische Symptome und klinische Diagnose}

Die häufigsten klinischen Symptome bei ZSCR sind in der Übersicht dargestellt. 


\section{Übersicht}

Die häufigsten klinischen Symptome der zentralen serösen Chorioretinopathie (ZSCR)

- Zentralskotom

- Metamorphopsien

- Farbsinnstörung

- Mikropsie

- Hypermetropisierung

- reduzierte Kontrastsensitivität

Es muss zwischen einer akuten und chronischen Form der ZSCR unterschieden werden.

\section{Akute ZSCR}

Die akute ZSCR ist weit häufiger als die chronische Form und präsentiert sich typischerweise mit Abhebung der neurosensorischen Netzhaut und Akkumulation von seröser Flüssigkeit zwischen dem retinalen Pigmentepithel (RPE) und Photorezeptoraußensegmenten. Der Visus ist meist relativ gut.

Bei der Fundusuntersuchung zeigt sich klassisch eine oval bis rundlich leicht dunkler erscheinende Elevation in der Makularegion, die der neurosensorischen Abhebung entspricht. Der foveale Reflex kann fehlen bzw. durch einen gelblichen Punkt, durch Sichtbarwerden des Xanthophylls, imponieren. Die seröse Flüssigkeit ist normalerweise klar, kann aber in bis zu 10\% der Fälle fibrinös grau-weiß erscheinen. Häufig (5-63\%) sind Pigmentepithelabhebungen (PEA) und Pigmentverklumpungen mit ZSCR assoziiert. Manchmal sind die PEA sehr groß und liegen außerhalb der neurosensorischen Abhebung oder kommen auch multipel vor.

Der größte Teil (> 50\%) der akuten ZSCR-Fälle bildet sich innerhalb von 2-3 Monaten spontan zurück, und die Sehkraft erholt sich meist vollständig.

Es hat sich aber gezeigt, dass der Visusanstieg vom Visus bei Erstüberprüfung abhängig ist. Je schlechter der Visus bei Erstpräsentation $(<0,7)$, desto schlechter ist die Wahrscheinlichkeit der vollständigen Visuserholung.

Nach Resorption einer ZSCR können RPE-Atrophien und Pigmentepithelverschiebungen eine altersabhängige Makuladegeneration (AMD) vortäuschen. Im Unterschied zur AMD sind diese Läsionen grundsätzlich stabil und ändern sich im Laufe der Zeit nicht.
Es ist nicht ungewöhnlich, dass nach einer Episode einer akuten ZSCR wieder eine ZSCR auftritt. Etwa ein Drittel bis die Hälfte der Patienten mit akuter ZSCR hat innerhalb von einem Jahr ein Rezidiv, während 10\% 3 oder mehr Rezidive innerhalb von 15 Jahren bekommen. Bei Rezidiven ist die neurosensorische Netzhautabhebung meist weniger bullös und die Visusprognose schlechter.

Reduziertes Kontrastsehen und geringe Metamorphopsien können nach Spontanresorption und wieder vollständigem Visus $(1,0)$ bei akuter ZSCR bei ca. $40 \%$ der Patienten zurückbleiben.

\section{Chronische ZSCR}

Die chronische Form der ZSCR betrifft ungefähr 5\% der Fälle und ist charakterisiert durch

- diffuse RPE-Pigmentveränderung,

- RPE-Atrophie,

- Pigmentverklumpungen und

- chronische flache seröse Netzhautabhebung.

Zum Zeitpunkt der Diagnosestellung sind die Patienten meist älter und weisen einen deutlich schlechteren Visus auf als Patienten mit akuter ZSCR. Die Sehverschlechterung ist oft permanent. Intraretinale Zysten scheinen mit einer längeren Abhebung der neurosensorischen Netzhaut assoziiert zu sein.

Es wird vermutet, dass ca. 16\% der akuten ZSCR-Fälle in eine chronische Form übergehen (Liew et al. 2013). Obwohl es schwierig ist, den Übergang genau zu definieren, wird im klinischen Alltag nach 6 Monaten ohne Heilungstendenz von einer chronischen Form gesprochen.

Eine choroidale Neovaskularisation kann nach einer ZSCR zwischen 0,3 und 2\% pro Jahr und Patient entstehen (Liew et al. 2013). Eine weitere Veränderung, die bei chronischer ZSCR auftritt, ist eine Verdünnung der neurosensorischen Retina, hauptsächlich der Photorezeptorschicht.

\section{Pathogenese}

Die Pathophysiologie der ZSCR ist noch immer nicht geklärt. Die meisten Untersucher glauben, die primäre Pathologie findet sich im RPE oder der Choriokapillarisschicht der Aderhaut oder beiden. 

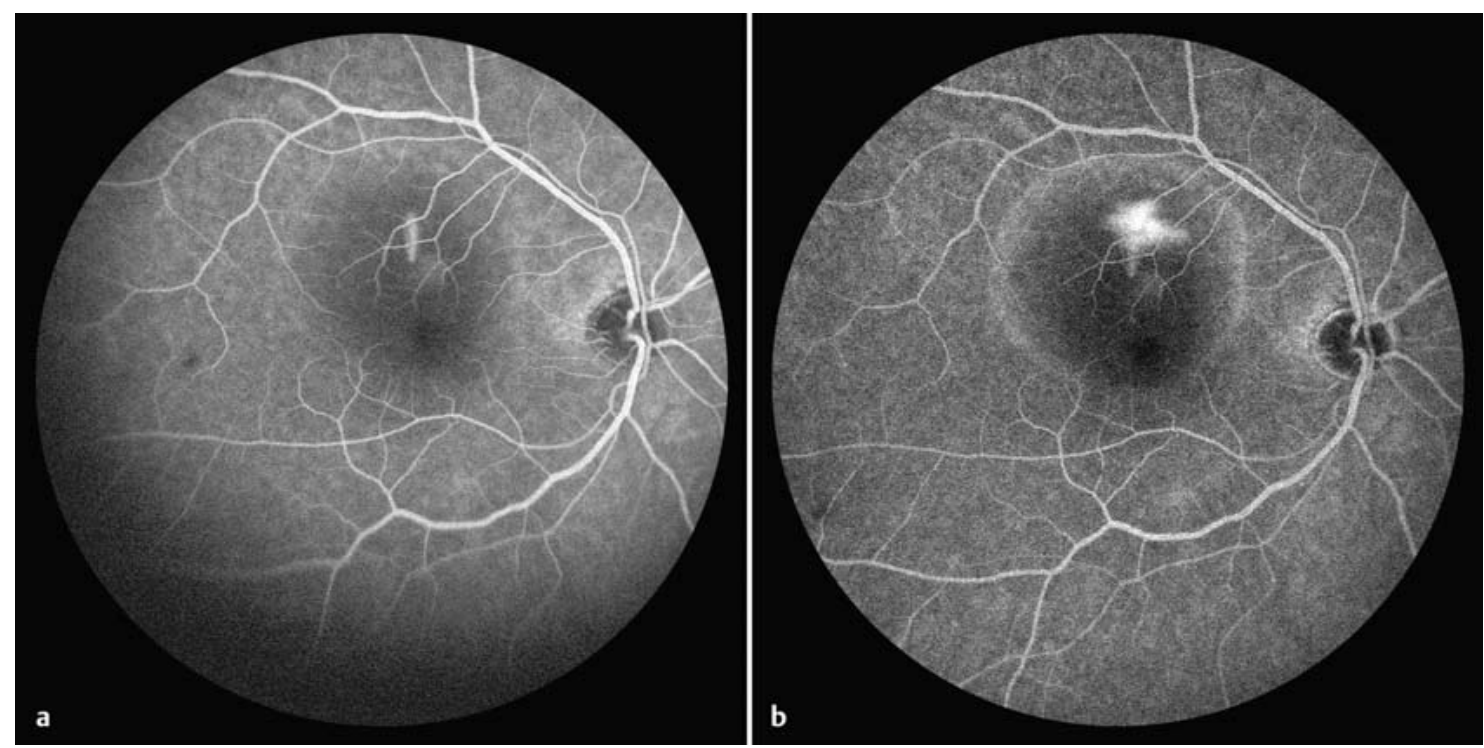

Abb. 1 Fluoresceinangiografie bei akuter zentraler seröser Chorioretinopathie (ZSCR). a Frühphase einer akuten ZSCR mit beginnendem „rauchenden Kamin“. b Spätphase einer akuten ZSCR mit „rauchendem Kamin“.

In der Fluoresceinangiografie können bei der akuten ZSCR ein oder mehrere RPE-Leckagepunkte, die meist extrafoveolär liegen, detektiert werden. Das typische Bild (7-20\%) wird als „rauchender Kamin“ (Abb. 1) oder „Tintenklecks“ beschrieben. Nichtsdestotrotz findet sich meist eine subfoveale neurosensorische $\mathrm{Ab}$ hebung, die auf die beginnende Atrophie bzw. die geringere Pumpfunktion des RPE zurückzuführen sein dürfte.

Die RPE-Dysfunktion kann zu einer fokalen oder diffusen/multifokalen akuten ZSCR führen. Die RPE-Dysfunktion scheint mehr als der reine RPE-Verlust eine Schlüsselrolle bei der Pathogenese einzunehmen.

In der Indocyaningrünangiografie zeigen sich aktive choroidale Leckagen. Man glaubt, dass die choroidale Hyperpermeabilität kleine PEA entstehen lässt, die einreißen und anschließend zur neurosensorischen Abhebung der Retina führen. Der ursprüngliche Grund für die choroidale Hyperpermeabilität ist jedoch ungeklärt, könnte aber mit der beeinträchtigten Autoregulation des choroidalen Blutflusses zusammenhängen. Diese Ansicht stammt von Untersuchungen, die zeigen, dass in Bereichen von choroidaler Ischämie Leckagen auftreten, die mit Bereichen gesteigerter Hyperpermeabilität assoziiert sind.

Die Störung der Autoregulation des choroidalen Blutflusses könnte auch die Verbindung von ZSCR und einem erhöhten Katecholaminspiegel, Sympathikus- aktivität und arterieller Hypertonie erklären. In SD-OCT-Untersuchungen konnte eine Zunahme der choroidalen Dicke im Enhanced-Depth-Imaging-Modul (EDI-Modul) bei ZSCR-Patienten von 50-80\% gemessen werden (Liew et al. 2013). Aber es gibt bis dato keine publizierte prospektive Studie, die eine erhöhte Inzidenz von ZSCR bei Menschen mit dickerer Aderhaut beschreibt.

Die Pathophysiologie der chronischen ZSCR dürfte sich etwas von der akuten Form unterscheiden. Die RPEDysfunktion führt zu einer diffusen und weitverzweigten granulären Hyperfluoreszenz in der Fluoreszenzangiografie. Wahrscheinlich spiegelt das die RPE-Atrophieareale nach seröser Netzhautabhebung wider. Identisch zeigen sich in der Indocyaningrünangiografie verzweigte Areale mit choroidaler Hyperpermeabilität.

Sehr hilfreich ist die Autofluorenszenzuntersuchung zur Unterscheidung einer akuten von einer chronischen ZSCR-Form. Bei der chronischen Form sieht man typischerweise eine hyperfluoreszente Umrandung der serösen Netzhautabhebung. Wahrscheinlich spielt dabei die Akkumulation von hyperfluoreszenten Quellpunkten eine große Rolle, die biomikroskopisch als gelbliche Ablagerungen imponieren. 


\section{Diagnostisches Vorgehen}

\section{- Bildgebende Verfahren}

Die optische Kohärenztomografie (OCT) hat sowohl bei der Diagnosestellung als auch bei der Verlaufsbeurteilung eine wichtige Stellung eingenommen. Kleinste biomikroskopisch nicht genau identifizierbare Veränderungen wie subretinale Flüssigkeit, Pigmentepithelabhebungen (PEA) und RPE-Atrophien können gut dargestellt werden (Abb. 2). Eine Dickenzunahme bzw. -abnahme der Choroidea kann mit dem EDI-Modul bestimmt werden.

Die Fluorescein- bzw. Indocyaningrünangiografie bieten eine weitere Möglichkeit, eine ZSCR von anderen Pathologien zu unterscheiden.

Die Fundusautofluoreszenzuntersuchung ist durch die Lipofuscinablagerung im RPE erklärbar. Diese nicht invasive Methode ist gut geeignet, um chronische Verläufe zu beobachten.

Nahinfrarot- und Fundusautofluoreszenz könnten in Zukunft zur Darstellung von Fluorophoren wie A2E, die sich in den Lipofuscingranula befinden, genutzt werden.

\section{- Funktionelle Untersuchungen}

Das multifokale Elektroretinogramm (mfERG) bzw. die Mikroperimetrie spielen bei der Diagnosestellung der ZSCR eine untergeordnete Rolle. In Bereichen von resorbierter subretinaler Flüssigkeit zeigen sich häufig ein verändertes mfERG bzw. reduzierte Sensitivität in der Mikroperimetrie.

\section{Therapeutisches Vorgehen}

Bei der akuten ZSCR-Form bildet sich ein Großteil der Fälle spontan zurück.

Die exogene Zufuhr von Kortikosteroiden sollte möglichst vermieden bzw. zumindest reduziert werden, und andere Risikofaktoren wie psychosozialer Stress

\section{Prinzipien}

Therapeutische Prinzipien bei zentraler seröser Chorioretinopathie (ZSCR)

- Reduzierung von Risikofaktoren

- fokale Lasertherapie

- photodynamische Therapie (PDT)

- Diodenlaser, Mikropulsdiodenlaser, transpupillare Thermotherapie (TTT)

- Anti-vascular-endothelial-Growth-Factor-Medikamente (Anti-VEGF-Medikamente)

- andere Medikamente (Kortikosteroidinhibitoren, Adrenalinrezeptorinhibitoren)

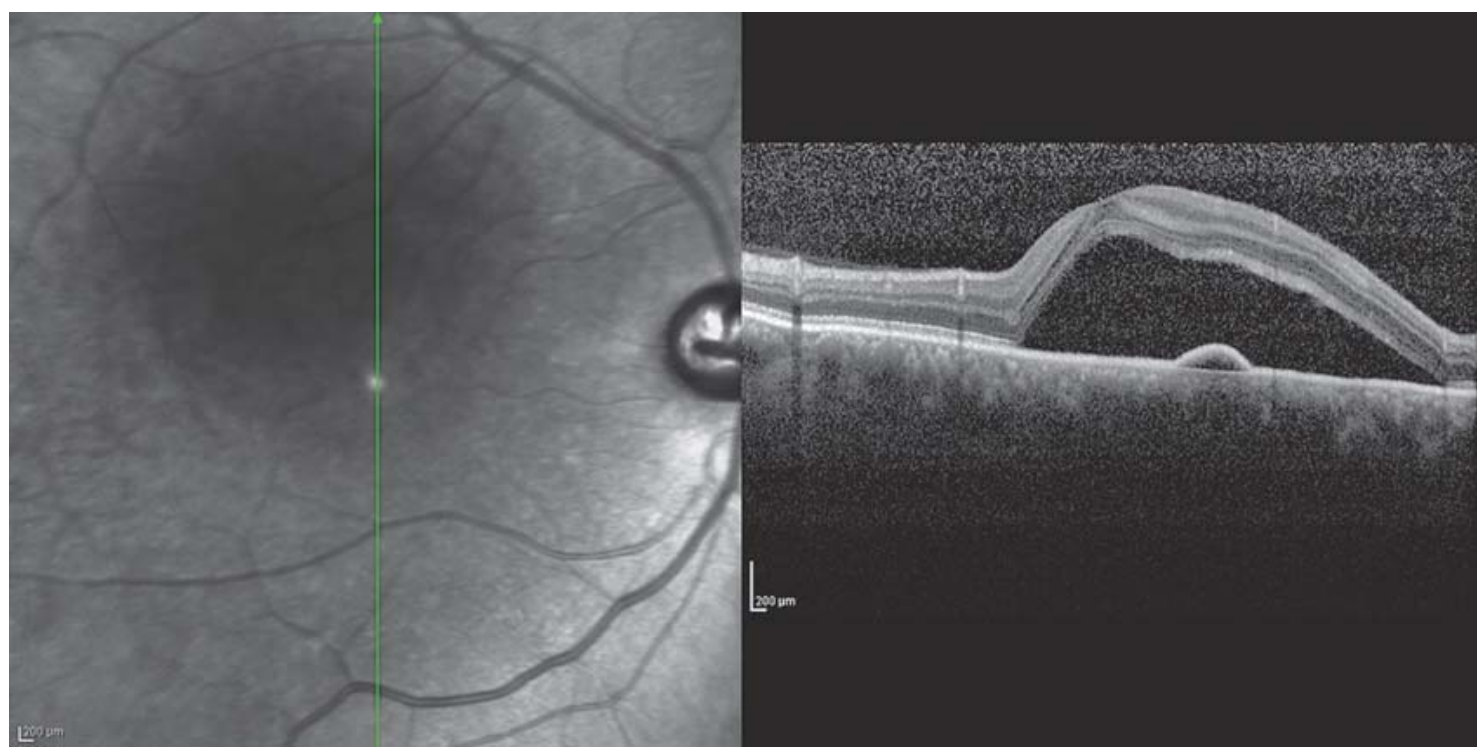

Abb. 2 OCT-Aufnahme einer akuten ZSCR. 
sollten abgebaut werden. Dennoch ist die Evidenz sehr gering, dass diese Maßnahmen den natürlichen Verlauf der Erkrankung beeinflussen.

\section{- Fokaler Argonlaser}

Wenn sich die seröse Netzhautablösung nach 3-4 Monaten nicht spontan resorbiert, können kleine $(250 \mu \mathrm{m})$ extrafoveale Leckagen mittels Argonlaserphotokoagulation ( 2 - 5 Spots, $100-200 \mu \mathrm{m}, 100-200 \mathrm{~mW}$, $100 \mathrm{~ms}$ ) behandelt werden, indem eine kleine fibrotische Narbe erzeugt wird. Laserspotgrößen $<100 \mu \mathrm{m}$ haben ein erhöhtes Risiko für die Entwicklung einer choroidalen Neovaskularisation (CNV).

Auch andere Komplikationen wie permanente Skotome und foveale Narben wurden beschrieben, sodass primär zumindest 3 Monate zugewartet werden sollte. Langzeitbeobachtungen haben jedoch keinen Vorteil in Bezug auf Endvisus, Farbsinnstörung, Rezidivhäufigkeit und Progression in eine chronische Form gesehen (Nicholson et al. 2013, Quin et al. 2013). Des Weiteren muss darauf geachtet werden, dass der Patient ausführlich über mögliche Komplikationen einer fokalen Lasertherapie aufgeklärt wird.

\section{- Photodynamische Therapie (PDT)}

Mit der photodynamischen Therapie können subfoveal oder juxtafoveal gelegene Leckagen, multifokale Leckagen und choroidale Anomalien behandelt werden.

Die Behandlung führt zur Verdünnung der Choriokapillaris und zu choroidaler Hypoperfusion. Nebeneffekte sind Pigmentveränderungen im Behandlungsareal, RPE-Verlust, sekundäre CNV und chronisch choroidale Hypoperfusion. Darüber hinaus muss beachtet werden, dass subretinales Fibrin an Verteporfinmoleküle binden könnte und so zur gesteigerten Energieabsorption mit früheren Komplikationen bei Standard-PDT-Behandlungen führen könnte.

Behandlungen mit einer Full-Dose- (6 mg/m² Körperoberfläche) Standard-PDT ( $\left.50 \mathrm{~J} / \mathrm{cm}^{2}\right)$ führte in den meisten ZSCR-Fällen zur Resorption der serösen Flüssigkeit. Es gibt aber auch Berichte von plötzlichem Sehverlust, schwerer choroidaler Ischämie, RPE-Atrophie und choroidalen Neovaskularisationen nach StandardPDT. Half-Dose-PDT ( $3 \mathrm{mg} / \mathrm{m}^{2}$ Körperoberfläche) hatte einen stabilen bzw. sogar besseren Visus zur Folge. Des Weiteren wurden mit Half-Fluence-Behandlung $\left(25 \mathrm{~J} / \mathrm{cm}^{2}\right)$-Behandlungen gleiche Ergebnisse in Bezug auf Visusanstieg und Resorption der subretinalen Flüssigkeit beschrieben verglichen mit Full-Fluence-Behandlungen.

Es gibt keine Vergleichsstudien zwischen Half-Doseund Half-Influence-Behandlungen. Ebenso wenig ist es klar, ob weitere Veränderungen anderer PDT-Parameter zur erfolgreichen Behandlung einer ZSCR führen.

Besonders gut auf PDT-Behandlungen haben Patienten mit chronischer ZSCR und Hyperfluoreszenz im ICG angesprochen.

\section{- Diodenlaser, Mikropulsdiodenlaser, transpupillare Thermotherapie (TTT)}

Verglichen mit dem Argonlaser hat der Diodenlaser eine tiefere Eindringtiefe und erreicht die Choroidea, das mögliche pathologische Korrelat. Studien haben gezeigt, dass der Visusanstieg nach 4 Wochen besser war als nach Argonlaserbehandlung, jedoch nach 8 Wochen bezüglich des Visusanstieges kein Unterschied mehr zu finden war (Quin et al. 2013). Im Vergleich zum Argonlaser gibt es keinen Anhalt dafür, dass der Mikropulsdiodenlaser einen Vorteil bei der Behandlung eines Leckagepunktes bei ZSCR bietet.

Es gibt kleinere Studien, die eine teilweise erfolgreiche Therapie mit Mikropulsdiodenlaser von chronischer ZSCR mit juxtafoveolären Leckagen in der Fluoreszenzangiografie zeigen (Quin et al. 2013). Auch eine Behandlung mit TTT eignet sich zur Behandlung von juxtafoveolären Leckagen. Dennoch sind zur Beurteilung dieser Behandlungsoptionen noch weitere Studien notwendig, um feststellen zu können, ob gegenüber einer PDT oder fokalen Lasertherapie ein Vorteil besteht.

\section{Anti-VEGF-Medikamente}

Es ist nach wie vor ungeklärt, wie Anti-VEGF-Medikamente bei ZSCR wirken, da ja keine choroidalen Neovaskularisationen vorhanden sind. Untersuchungen zeigen, dass im Vergleich zwischen monatlichen intravitrealen Anti-VEGF-Medikamentenapplikationen und einmaliger Standard-Fluence-PDT der Endvisus gleich ist. Dennoch muss hervorgehoben werden, dass die Visusverbesserung bei PDT-Behandelten früher eintrat. 
Die Applikation von Anti-VEGF-Medikamenten soll bei jenen ZSCR-Fällen eine Rolle spielen, in denen subfoveolär eine Akkumulation von Fibrin stattgefunden hat. Hier soll die PDT eher zurückhaltend angewendet werden.

Kombinationstherapien mit PDT haben in kleinen retrospektiven Fallstudien gezeigt, dass die subretinale Flüssigkeit schneller resorbiert wird. Aber es haben sich nach einigen Monaten retinale Atrophien entwickelt. Der Mechanismus, wie Anti-VEGF auf die choroidalen Gefäße bei ZSCR wirkt, ist aber nach wie vor ungeklärt.

\section{Andere Medikamente}

Spironolacton (25 mg oral $2 \times$ täglich) wurde in kleineren Studien zur Behandlung der ZSCR eingesetzt. Es zeigte sich zwar eine Abnahme der subretinalen Flüssigkeit, der Visusanstieg war aber eher gering. Nach Absetzen des Spironolactons nimmt die subretinale Flüssigkeit meist wieder schnell zu (Herold et al. 2014).

Eine kleine Studie mit dem Glukokortikoidrezeptorblocker Mifepriston (RU486) hat einen Visusanstieg durch Besserung des retinalen zystischen Ödems gezeigt (Quin et al. 2013).

Orale Betablocker (z. B. Propanolol) sind nur bei wenigen Patienten zur Behandlung einer chronischen ZSCR eingesetzt worden. Es zeigten sich eine Resorption von subretinaler Flüssigkeit und ein Visusanstieg (Quin et al. 2013).
Cave. Der Augenarzt sollte allerdings systemische Medikamente aufgrund von systemischen Nebenwirkungen sehr ausgewählt einsetzen.

\section{Grubenpapille}

\section{Definition}

Die Grubenpapille ist eine meist einseitige, angeborene Papillenanomalie, die mit einer Häufigkeit von 1: 10000 auftritt. Funduskopisch zeigt sich eine rundliche bis ovale gräulich erscheinende Vertiefung, typischerweise am temporalen Papillenrand. In fast der Hälfte der Fälle entwickeln sich im jungen Erwachsenenalter sekundäre Makulaveränderungen mit Visusminderung.

\section{Klinisches Bild}

Der Patient beschreibt meist ein Zentralskotom oder Metamorphopsien.

Die Makulopathie kann sich präsentieren als

- Makulaödem in verschiedenen Schichten der Retina

- Makulaforamen

- seröse Makulaabhebung (Abb. 3)

- epiretinale Gliose

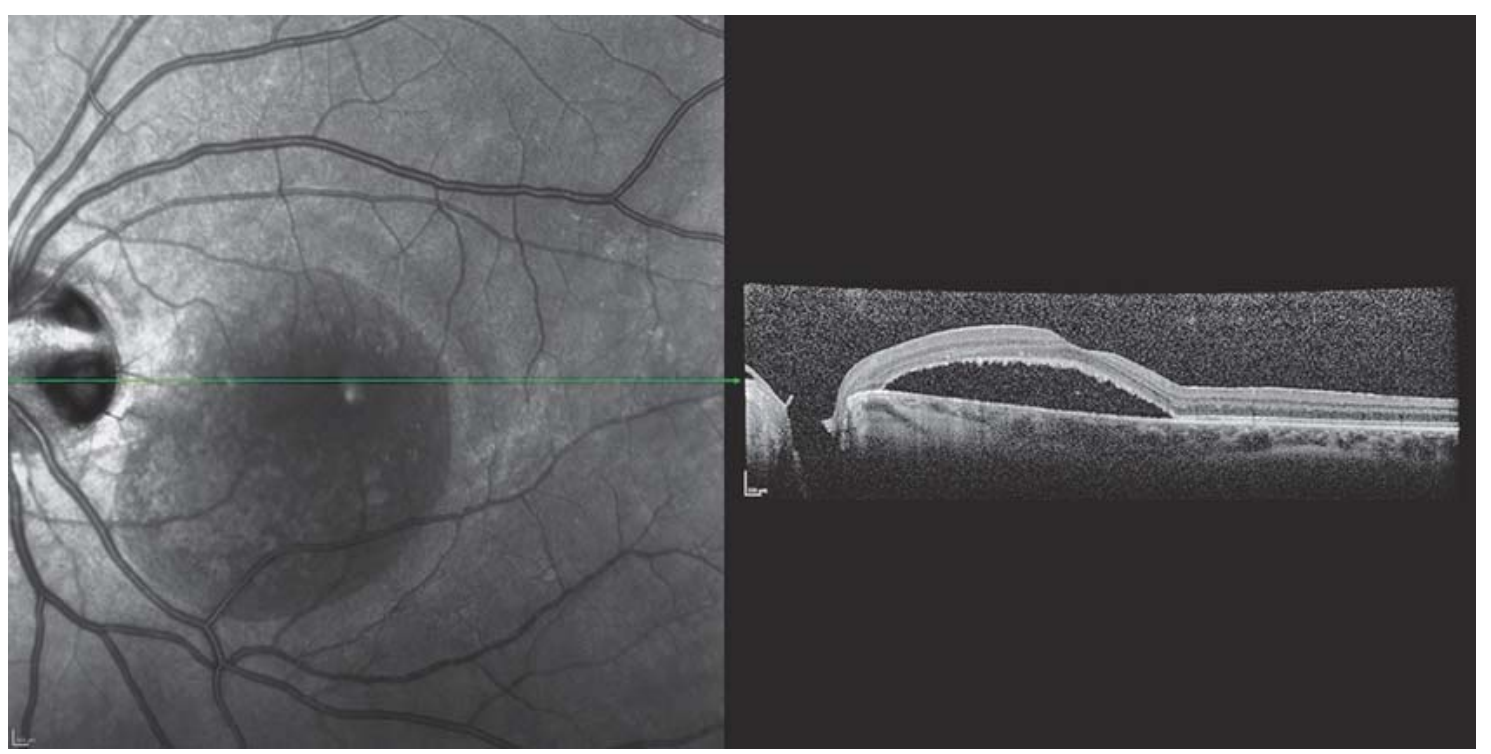

Abb. 3 OCT-Aufnahme einer Grubenpapille mit seröser Makulaabhebung. 


\section{Pathogenese}

Fehlbildungen von normalen anatomischen Strukturen an der Papille führen dazu, dass Liquor und Glaskörper entlang eines Druckgradienten in die verschiedenen Netzhautschichten bzw. in den subretinalen Raum eindringen. Es wird diskutiert, dass eine hintere Glaskörperabhebung und/oder ein translaminarer Druckgradient für die Bildung einer sekundären Makulopathie bei der Grubenpapille mitverantwortlich ist.

\section{Diagnostisches Vorgehen}

Durch eine gründliche Fundusuntersuchung kombiniert mit einer OCT-Untersuchung ist eine schnelle Diagnosestellung möglich.

\section{Therapeutisches Vorgehen}

Aufgrund der nicht ganz gesicherten Pathogenese gibt es auch verschiedene Therapien, über deren Erfolg sehr unterschiedlich berichtet wird (s. Infobox „Prinzipien“).

\section{Prinzipien}

Therapeutische Prinzipien bei Grubenpapille

- abwartende Haltung

- orales Acetazolamid oder Kortikosteroide

- juxtapapilläre Laserkoagulation

- Pars-plana-Vitrektomie mit Gaseingabe

Bei noch gutem Visus ist eine zunächst abwartende Haltung (2-3 Monate) bei spontaner Rückbildung in ca. 25\% der Fälle oder ein pharmakologischer Therapieansatz sinnvoll. Orales Acetazolamid oder Kortikosteroide senken den intrakraniellen Druck. Es kommt aber nach Absetzen des Medikaments häufig zu Rezidiven, sodass eine Kombination mit einem operativen Vorgehen überlegt werden sollte. Eine juxtapapilläre temporale Laserkoagulation mit einer Spotgröße von $100-200 \mu \mathrm{m}$ in 3-4 Reihen mit moderater Intensität führt zu einer Barriere für den subretinalen Raum, dennoch ist die Laserkoagulation allein meist von enttäuschendem Erfolg und wird deshalb häufig mit einer Pars-plana-Vitrektomie mit oder ohne Gastamponade kombiniert, um auch den intraretinalen Flüssigkeitsstrom zu blockieren. Dies führt in ca. zwei Drittel der Fälle zu einem deutlichen Visusanstieg.

\section{Myope Makulopathie}

Myopie ist mit einem Anteil von 15-40\% der Bevölkerung die häufigste Anomalie des Auges, und die Zahl der Betroffenen steigt an. Es gibt ausgeprägte geografische Unterschiede mit der größten Prävalenz in Asien und der geringsten in Afrika. Im Gegensatz zur Myopie ist die pathologische Myopie (PM; Synonyme: Myopia alta, degenerative Myopie) selten, da sie nur etwa $10 \%$ der Kurzsichtigen betrifft. Dies entspricht einer Prävalenz von ca. $2 \%$ bezogen auf die Gesamtbevölkerung. Die myope CNV (Abb. 4) ist eine visusbedrohende Komplikation, die 5-10\% der von PM Betroffenen entwickeln.

\section{Pathophysiologie der PM}

PM wird definiert durch eine Refraktion von mehr als - 6,0 dpt sphärischem Äquivalent und einer Achslänge von über 26,5 mm. Über diese Grenzen gibt es noch keinen Konsens, daher werden sie von verschiedenen Autoren auch teilweise anders gesetzt. Durch das große Längenwachstum des Augapfels kommt es zu einer Dehnung von Sklera, Aderhaut und Netzhaut sowie einem veränderten Verhalten des Glaskörpers.

Für die Entstehung einer myopen Makulopathie sind insbesondere die Verdünnung der Aderhaut und eine geringere Dichte des retinalen Pigmentepithels, die zu einer im Vergleich mit dem emmetropen Auge ungünstigeren Sauerstoffversorgung der Makula führen (Benavente-Pérez et al. 2010), verantwortlich. Starkes Längenwachstum und dünne Sklera begünstigen die Entstehung von Staphylomen.

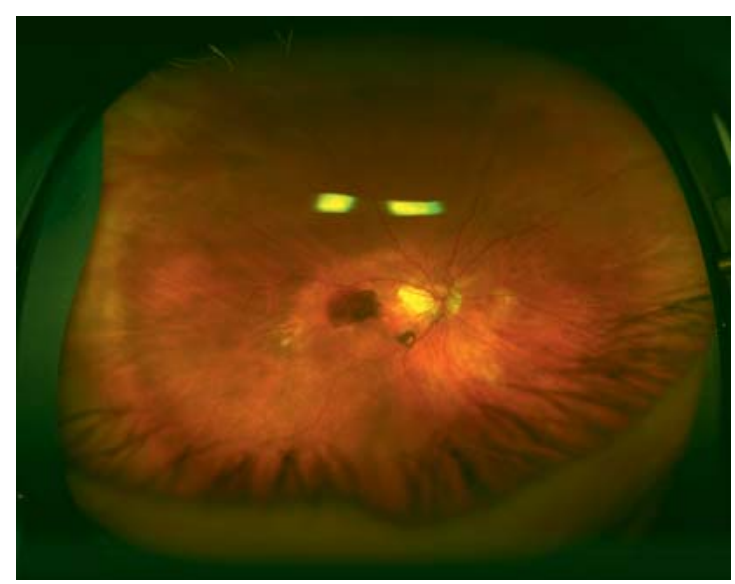

Abb. 4 Weitwinkelfundusaufnahme bei myoper CNV mit Blutung. 
Bei großen Achslängen kommt es zu einem etwa um 10 Jahre früheren Auftreten einer hinteren Glaskörperabhebung im Vergleich zu normal langen Augen. Kommt es nicht zu einer Abhebung, kann eine Vitreoschisis entstehen, durch die eine myope Makuloschisis verursacht werden kann.

Das Auftreten einer myopen CNV ist in allen Stadien der myopen Makulopathie (Tab. 1) möglich. Es kommt häufig zu einem Fortschreiten der Makulopathie, die Wahrscheinlichkeit einer Progression steigt insbesondere durch die Präsenz von Lacksprüngen auf 70\%, während sie in den ersten Stadien lediglich $10 \%$ beträgt.

\section{Myope CNV}

Myope CNV ist die häufigste CNV bei unter 50-Jährigen und führt daher im Gegensatz zu CNV im Rahmen einer AMD zu Krankenständen und Arbeitsunfähigkeit.

\section{Diagnostisches Vorgehen}

Aufgrund der meist vorbestehenden Pathologien an der Makula ist die graue, subretinale Membran, typischerweise mit einem Durchmesser von unter $1000 \mu \mathrm{m}$, biomikroskopisch oft schwierig zu erkennen. In über der Hälfte der Fälle ist sie subfoveal lokalisiert.

Auch die SD-OCT-Untersuchung (Abb. 5) ist bei PM zur Diagnosestellung einer CNV nur bedingt geeignet, da sie sich schlecht von einer Makuloschisis differenzieren lässt.

\section{Tabelle 1}

Stadieneinteilung von myoper Makulopathie (Avila et al. 1984)

\begin{tabular}{|c|c|}
\hline Stadium & Kennzeichen \\
\hline M0 & - unauffälliger hinterer Pol \\
\hline M1 & $\begin{array}{l}\text { - Pigmentarmut } \\
\text { - Tessellation (Sichtbarkeit von Aderhautgefäßen) }\end{array}$ \\
\hline M2 & $\begin{array}{l}\text { - Pigmentarmut } \\
\text { - Tessellation } \\
\text { - hinteres Staphylom }\end{array}$ \\
\hline M3 & $\begin{array}{l}\text { - Pigmentarmut } \\
\text { - Tessellation } \\
\text { - hinteres Staphylom } \\
\text { - Lacksprünge }\end{array}$ \\
\hline M4 & $\begin{array}{l}\text { - Pigmentarmut } \\
\text { - Tessellation } \\
\text { - hinteres Staphylom } \\
\text { - Lacksprünge } \\
\text { - fokale Aderhautatrophien }\end{array}$ \\
\hline M5 & - große Areale geografischer Aderhautatrophie am hinteren Pol \\
\hline
\end{tabular}

Somit bleibt die Fluoresceinangiografie (Abb. 6) die wichtigste Untersuchung zur Diagnosestellung, die in jedem Fall bei Präsenz von sub- oder intraretinaler Flüssigkeit durchgeführt werden sollte.

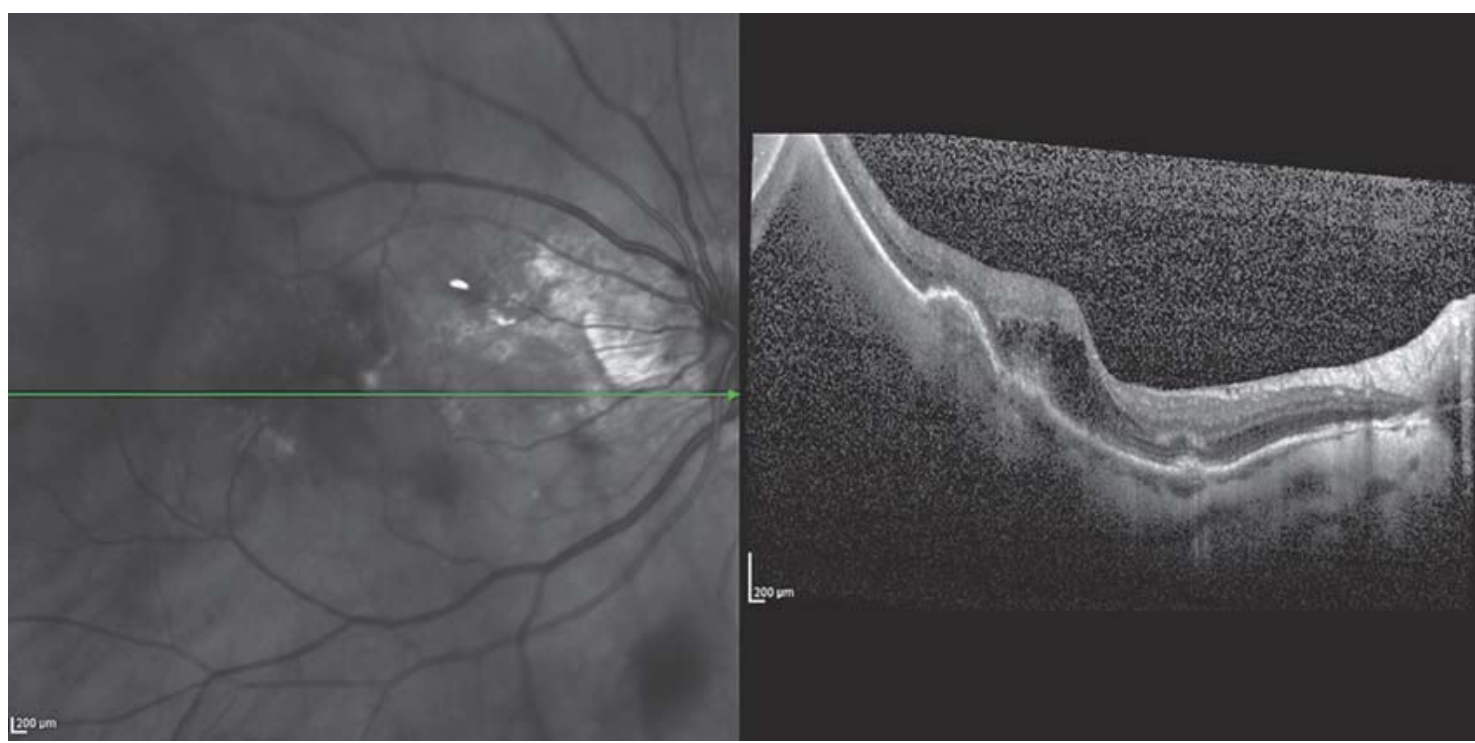

Abb. 5 OCT-Aufnahme bei myoper CNV. 


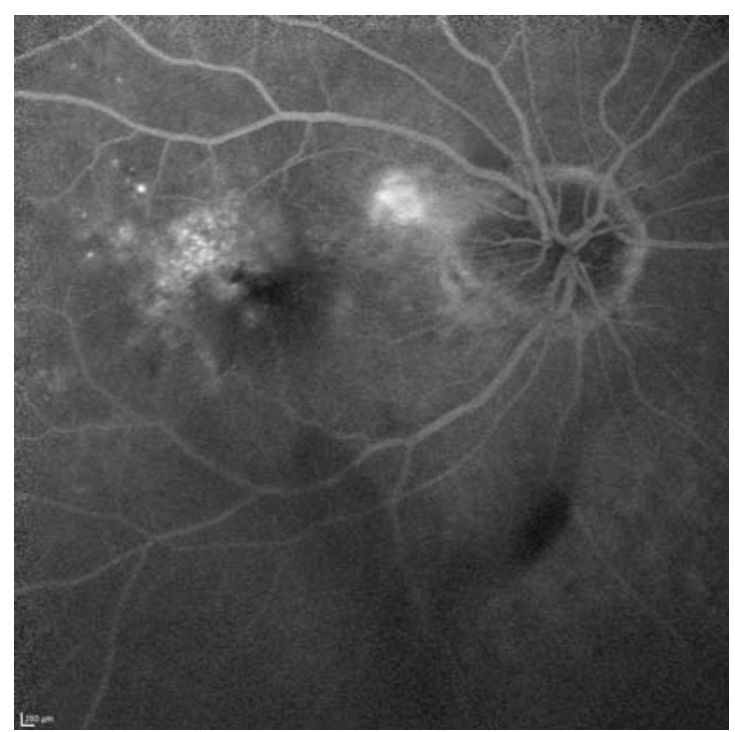

Abb. 6 Fluoresceinangiografie (FAG) in der Spätphase bei myoper CNV.

\section{Therapeutisches Vorgehen}

Früher wurde die Laserkoagulation von extrafovealen Läsionen durchgeführt, sie führte jedoch zu atrophen Narben, die sich bei der Narbenbildung noch ausdehnten. Daher kommt sie heute bei juxta- und subfovealen Läsionen nicht mehr in Betracht.

In der VIP-Studie (VIP report No. 1, 2001) konnte gezeigt werden, dass durch die photodynamische Therapie mit Verteporfin (vPDT) eine Visusstabilisierung über 24-36 Monate möglich ist. Daher wurde die PDT mit Verteporfin als Therapie der myopen CNV zugelassen.

Es gibt seit Beginn der Anti-VEGF-Ära in der neovaskulären AMD einige Studien, die Visusverbesserungen unter Anti-VEGF-Therapie zeigten (Silva 2012). Deshalb wurde die myope CNV von vielen Augenärzten Off Label mit Bevazicumab (Avastin) oder Ranibizumab (Lucentis) behandelt. Ende 2013 erfolgte die Zulassung von Ranibizumab (Lucentis) durch die EMA für diese Indikation.

Die Zulassungsstudie RADIANCE wurde im März 2014 publiziert (Wolf et al. 2014). Diese prospektive Studie besteht aus 3 Armen:

- In der 1. Gruppe erhielten die Patienten 0,5 mg Ranibizumab intravitreal am Tag 1 und nach 1 Monat, danach je nach Visus.
- In der 2. Gruppe erhielten die Patienten 0,5 mg Ranibizumab intravitreal am Tag 1 , danach je nach OCT-Befund.

- Die 3. Gruppe erhielt vPDT am Tag 1 und nach 3 Monaten nach Entscheidung des Untersuchers neuerliche vPDT oder 0,5 mg Ranibizumab intravitreal.

In den Gruppen 1 und 2 wurde in den ersten 3 Monaten eine Visussteigerung von über 2 Zeilen erzielt versus Gewinn von 1,4 Buchstaben in der 3. Gruppe. Dieser Unterschied ist hochsignifikant, und der Gewinn blieb bis zum 12. Monat erhalten. Die Patienten erhielten im Schnitt 4,0, 2,0 und 2,0 Injektionen, wobei in der 3. Gruppe $27 \%$ der Patienten keine Injektion bekamen. Gruppe 2 mit der SD-OCT-gesteuerten Wiederbehandlung benötigte weniger Injektionen bei gleich gutem Visusergebnis nach 12 Monaten.

In Asien wurde eine Phase-III-Studie mit Aflibercept (Eylea) durchgeführt, deren Ergebnisse aber noch nicht publiziert sind.

\section{Verlaufskontrolle}

Entsprechend einer Stellungnahme der Deutschen Ophthalmologischen Gesellschaft vom März 2014 (Pauleikoff et al. 2014) sollte nach einmaliger intravitrealer Verabreichung von Ranibizumab (Lucentis) die Läsionsaktivität ein halbes Jahr lang monatlich mit Visuskontrolle, Funduskopie und SD-OCT erfolgen, anschließend verlaufsabhängig in längeren Intervallen.

\section{Aderhautfalten}

Die Erstbeschreibung von Aderhautfalten (Striae) geht auf Nettleship 1884 zurück, der sie bei einem Patienten mit Papillenödem feststellte. In der Folge wurden Fälle von Aderhautfalten publiziert, die aufgrund eines orbitalen Tumors auftraten. Nachfolgende Arbeiten aber zeigten, dass auch ohne orbitale Druckerhöhung nur aufgrund skleraler Kontraktionen solche Veränderungen entstehen können. 


\section{Pathomechanismus}

Theoretisch betrachtet ist also neben der häufigen Genese aufgrund eines Druckes auf den Bulbus von außen, wie er typischerweise bei Tumoren der Sklera und der Orbita und benachbarter Strukturen wie Tumoren der Nasennebenhöhlen und Kieferhöhlen auftritt, auch die Genese aufgrund von Traktionen wie beispielsweise bei posterioren Skleritiden (dazu s. u. Fallbeispiel) möglich, die durch die Verdickung und Schrumpfung der Sklera zur Verformung der Aderhaut führen können. Dabei handelt es sich um einen ähnlichen Pathomechanismus hervorgerufen durch Traktionen, die auf die Aderhaut wirken, wie er typischerweise bei Aderhauttumoren oder - wie von Bird u. Sanders 1973 beschrieben - länger bestehenden Papillenödemen (hervorgerufen durch intrakranielle und extrakranielle Raumforderungen oder intrakranielle Druckerhöhung im Rahmen von Stenosen im Liquorsystem oder Blutungen) auftritt. Daneben kann aber auch ein Druckabfall im Inneren des Bulbus, wie er beispielsweise nach Verletzungen und nach operativen Eingriffen auftritt, zur Bildung von Aderhautfalten führen.

\section{Diagnostisches Vorgehen}

Wertvolle klinische Hinweise erhält man je nachdem, ob die Aderhautfalten unilateral oder bilateral auftreten. Bilaterale Aderhautfalten finden sich, wenn intrakranielle Druckerhöhungen zum Papillenödem führen. Unilaterales Auftreten weist auf Ursachen in der un- mittelbaren Umgebung des betroffenen Auges oder im Auge selbst hin, wobei im Auge selbst vor allem die neovaskuläre AMD und die Hyperopie Aderhautfalten induzieren können.

Die Anordnung der Falten verläuft zumeist in einem parallelen Muster schräg von der Papille weg in die Makula hinein oder auch radiär um die Papille verlaufend (Abb. 7).

Zu Symptomen kommt es eher selten. Der Patient bemerkt nur Metamorphopsien und damit einhergehend unter Umständen auch eine Sehverschlechterung, wenn die Falten die zentrale Makula betreffen.

\section{Therapeutisches Vorgehen}

Im Fall der Diagnose von Aderhautfalten oder des Verdachts auf Vorliegen von Aderhautfalten sollte nach Diagnosesicherung mittels Fluoresceinangiografie in allen Fällen eine bildgebende Abklärung mittels CT oder MRI erfolgen, um eine Raumforderung auszuschließen. Anschließend richtet sich die Therapie nach der Ursache der Erkrankung.

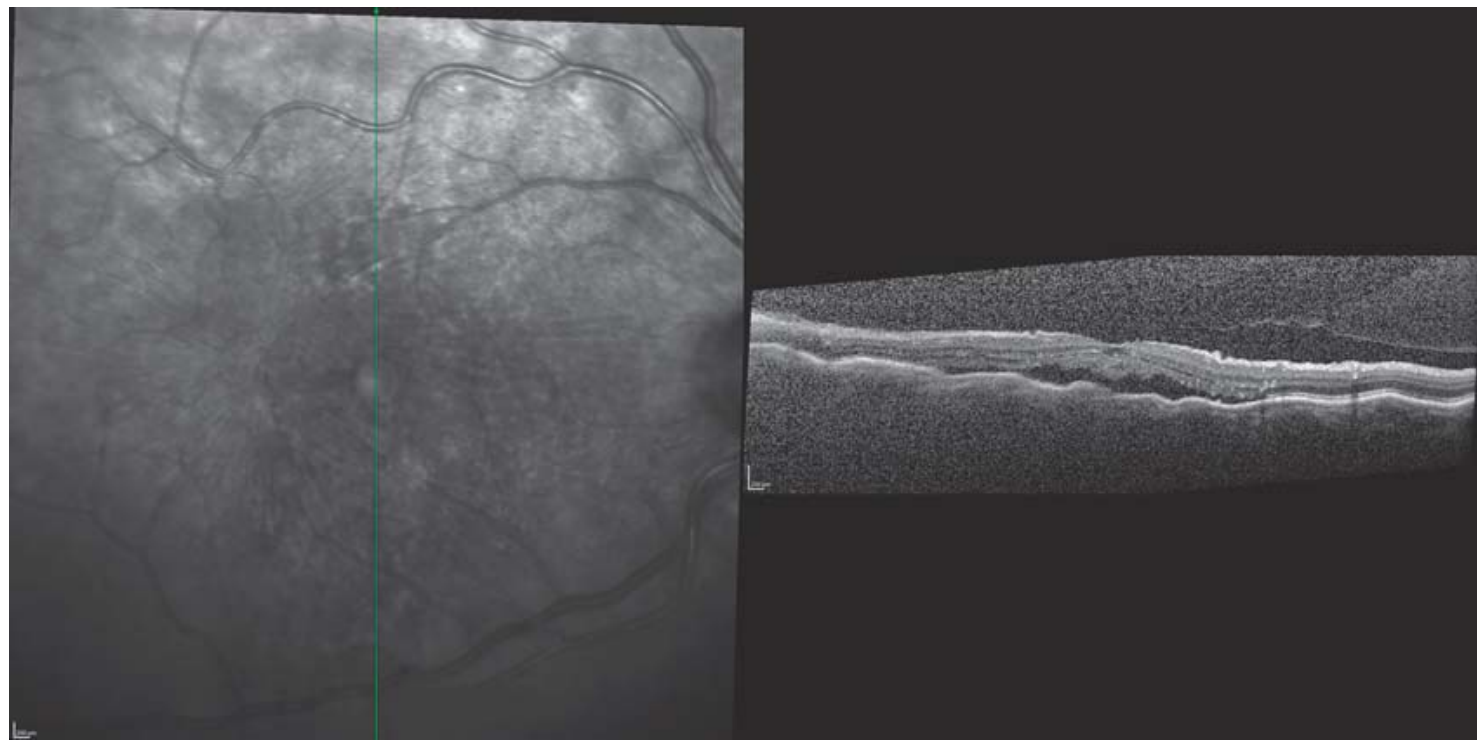

Abb. 7 SD-OCT-Aufnahme bei Aderhautfalten. 


\section{Kasuistik}

Fallbeispiel: Patientin mit Aderhautfalten

\section{Anamnese}

Eine 66-jährige Patientin stellt sich aufgrund einer Sehverschlechterung am rechten Auge vor. Die Patientin befindet sich wegen einer Polymyalgia rheumatica und Vorhofflimmern in internistischer Betreuung.

\section{Diagnostik}

Bei der ophthalmologischen Durchuntersuchung zeigt sich eine Visusreduktion auf 0,2 c. c. am rechten Auge. In der OCT-Untersuchung zeigt sich ein Makulaund Papillenödem, und in der nachfolgenden Fluorescein-/Indocyaninangiografie imponieren Aderhautfalten in typischer schräger paralleler Anordnung sowohl in der frühen Phase (Abb. 8) als auch in der Spätphase. Des Weiteren können nach den vorliegenden Befunden als Ursache für das Ödem eine Makuladegeneration mit CNV, Diabetes und Gefäßverschluss ausgeschlossen werden.

\section{Therapie}

Aufgrund des Verdachts auf posteriore Skleritis bei Polymyalgia rheumatica wird mit einer oralen Kortisontherapie (Startdosis $40 \mathrm{mg} / \mathrm{d}$ ) begonnen.

\section{Outcome}

Nach 7 Tagen oraler Kortisontherapie zeigt sich ein eindrucksvoller Rückgang des Makulaödems anhand der SD-OCT-Untersuchung. Auch das Papillenödem hat sich fast vollständig zurückgebildet.

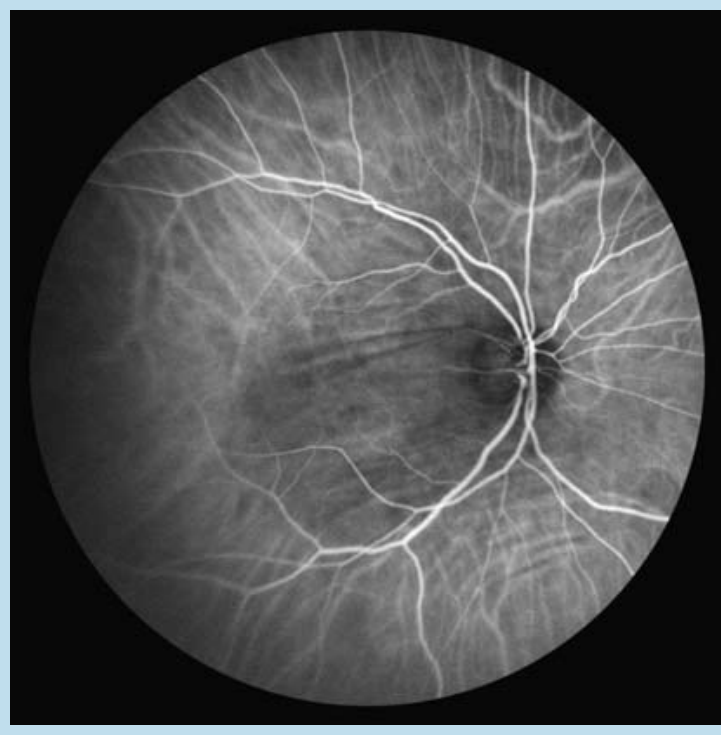

\section{Angioid Streaks}

\section{Definition}

Bei den - aus dem Englischen frei übersetzt - gefäßähnlichen Streifen handelt es sich um Veränderungen, die aufgrund von Degenerationen von elastischen Fasern in der Bruch-Membran entstehen.

\section{Diagnostisches Vorgehen}

Funduskopisch zeigen sich charakteristische, meist bilaterale rötliche bis bräunliche radiär von der Papille ausgehende Streifen, die im Rahmen von systemischen Erkrankungen wie Pseudoxanthoma elasticum, Morbus Paget, Sichelzellanämie, Akromegalie, Ehlers-DanlosSyndrom oder Diabetes mellitus oder aber auch idiopathisch auftreten können.

Erstmals beschrieben wurde die Erkrankung von Doyne 1889, und Knapp benannte sie 1892 mit der nun geläufigen Bezeichnung Angioid Streaks. Bock fand 1938 heraus, dass es sich nicht um eine Gefäßerkrankung handelt, wie bis zu diesem Zeitpunkt angenommen, sondern um Veränderungen in der Bruch-Membran, die aufgrund degenerativer Veränderungen von elastischen Fasern ausgelöst werden.

Meistens finden sich Angioid Streaks als Zufallsbefund, da sie keine Symptome per se hervorrufen. Nur wenn die Veränderungen die Makula betreffen und/oder zur Ausbildung einer submakulären choroidalen neovaskulären Membran (CNV) führen, kommt es zu einer Sehverschlechterung.

\footnotetext{
Normalerweise ist die Diagnose von Angioid Streaks allein mittels einer Funduskopie zu stellen, jedoch empfiehlt sich bei Verdacht auf eine makuläre Beeinträchtigung die Durchführung einer Fluoresczeinangiografie zur Unterscheidung, ob es sich um eine Makulaveränderung mit oder ohne Ausbildung einer sekundären CNV handelt.
}

Die Prognose der Angioid Streaks im Rahmen einer Sichelzellanämie ist besser als beim Ehlers-DanlosSyndrom, weil die Sichelzellanämie selten zur Ausbildung einer CNV führt, während im Gegensatz dazu das Vorliegen eines Ehlers-Danlos-Syndrom sehr häufig mit einer CNV einhergeht. 


\section{Therapeutisches Vorgehen}

Bei Vorliegen einer CNV sollte umgehend mit einer Anti-VEGF-Therapie begonnen werden, da in vielen Studien gezeigt werden konnte, dass sich bei frühem Therapiebeginn eine langanhaltende Stabilisierung der Sehkraft erreichen lässt. Verfahren, die vor der AntiVEGF-Therapie zur Anwendung kamen, wie beispielsweise die Laserkoagulation, die photodynamische Therapie oder die chirurgische Entfernung der CNV, führten demgegenüber zu keinen zufriedenstellenden Ergebnissen (dazu s.a. Stellungnahme der Retinologischen Gesellschaft, der Deutschen Ophthalmologischen Gesellschaft [DOG] und des Berufsverbands der Augenärzte Deutschlands zur Therapie choroidaler Neovaskularisationen bei Pseudoxanthoma elasticum (PXE); Stand März 2011).

Interessenkonflikt: Die Autoren geben an, dass sie in den letzten 3 Jahren Verbindungen zu den Firmen Novartis Pharma GmbH, Bayer AG, Allergan Inc., Hoya Vision Care Europe und Alcon Pharma GmbH hatten.

\section{Dieser Artikel erschien in den \\ Klinischen Monatsblättern für Augenheilkunde (DOI 10.1055/s-0033-1358018).}

\section{Literatur}

1 Avila MP, Weite JJ, Jalkh AE et al. Natural history of choroidal neovascularization in degenerative myopia. Ophthalmology 1984; 91: $1573-1581$

2 Benavente-Pérez A, Hosking SL, Logan NS et al. Ocular blood flow measurements in healthy human myopic eyes. Graefes Arch Clin Exp Ophthalmol 2010; 248: 1587-1594

3 Bird AC, Sanders MD. Choroidal folds in association with papilloedema. $\mathrm{Br}$ J Ophthalmol 1973; 57: 89-97

4 Connor PJ jr., Juergens JL, Perry $\mathrm{HO}$ et al. Pseudoxanthoma elasticum and angioid streaks. A review of 106 cases. Am J Med 1961; 30: $537-543$

5 Doyne RW. Choroidal and retinal changes. The results of blows on the eyes. Trans Ophthalmol Soc UK 1889; 9: 128

6 Fox A, Chan P, Lam D et al. Risk factors for recurrence of serous macular detachment in untreated patients with central serous chorioretinopathy. Ophthalmic Res 2011; 46: 160-163

7 Gass JD. Stereoscopic Atlas of macular Diseases. 3rd ed. St. Louis: Mosby; 1987: 102-109

8 Herold TR, Prause K, Wolf A et al. Spironolactone in the treatment of central serous chorioretinopathy - a case series. Graefes Arch Clin Exp Ophthalmol 2014; [Epub ahead of print]

9 Inhoffen W, Ziemssen F, Bartz-Schmidt KU. Chronische RCS (chronic Central Serous chorioretinopathy cCSC) als Differenzialdiagnose der feuchten, altersbedingten Makuladegeneration (AMD). Klin Monatsbl Augenheilkd 2012; 229: 889-896
10 Kalina RE, Mills RP. Acquired hyperopia with choroidal folds. Ophthalmology 1980; 87: 44-50

11 Kanski JJ, Milewski SA. Diseases of the Macula. London: Mosby; 2002: $55-56$

21 Kitzmann AS, Pulido JS, Diehl NN et al. The incidence of central serous chorioretinography in Olmsted County Minnesota, 1980 2002. Ophthalmology 2008; 115: $169-173$

12 Knapp H. On the formation of dark angioid streaks as unusual metamorphosis of retinal hemorrhage. Arch Ophthalmol 1892; 26: $289-292$

13 Lavinsky J, Lavinsky D, Lavinsky F et al. Acquired choroidal folds: a sign of idiopathic intracranial hypertension. Graefes Arch Ophthalmol Clin Exp Ophthalmol 2007; 245: 883-888

14 Liew G, Quin G, Gillies M et al. Central serous chorioretinopathy: a review of epidemiology and pathophysiology. Clin Exp Ophthalmol 2013; 41: $201-214$

15 Nicholson B, Noble J, Forooghian F et al. Central serous chorioretinopathy: update on pathophysiology and treatment. Surv Ophthalmol 2013; 58: $103-126$

16 Pauleikoff D, Bertram B, Claessens D. Stellungnahme der Deutschen Ophthalmologischen Gesellschaft, der Retinologischen Gesellschaft und des Berufsverbandes der Augenärzte Deutschlands zur Therapie choroidaler Neovaskularisationen bei Myopie. Ophthalmologe 2014; 111: 229-234

17 Quin G, Liew G, Ho I et al. Diagnosis and interventions for central serous chorioretinopathy: review and update. Clin Exp Ophthalmol 2013; 41: $187-200$

18 Silva R. Myopic maculopathy: A review. Ophthalmologica 2012; 228: $197-213$

22 Verteporfin in Photodynamic Therapy Study Group. Photodynamic therapy of subfoveal choroidal neovascularization in pathologic myopia with verteporfin. 1-year results of a randomized clinical trial - VIP report No. 1. Ophthalmology 2001; 108: $841-852$

19 Weissgold DJ, Brucker AJ. Chorioretinal Folds. In: Guyer DR, Yannuzzi LA, Chang S, Shields JA, Green WR, eds. Retina - Vitreous Macula. London: Saunders; 1999: 256-268

20 Wolf S, Balciuniene VJ, Laganovska G et al. RADIANCE: A randomized controlled study of Ranibizumab in patients with choroidal neovascularization secondary to pathologic myopia. Ophthalmology 2014; 121: 682-692

\section{Korrespondenzadresse}

Prof. Nikolaos E. Bechrakis

Universitätsklinik für Augenheilkunde und Optometrie

Medizinische Universität Innsbruck

Anichstraße 35

A-6020 Innsbruck

Österreich

Telefon: + 43-512/504 23720

Fax: + 43-512/504 23722

E-Mail: nikolaos.bechrakis@i-med.ac.at 


\section{CME.thieme.de}

\section{CME-Fragen}

\section{CME-Teilnahme}

- Viel Erfolg bei lhrer CME-Teilnahme unter http://cme.thieme.de

- Bitte informieren Sie sich über die genaue Gültigkeitsdauer unter http://cme.thieme.de

- Sollten Sie Fragen zur Online-Teilnahme haben, unter http://cme.thieme.de/hilfe finden Sie eine ausführliche Anleitung.

\section{1}

Wodurch ist die zentrale seröse

Chorioretinopathie (ZSCR)

gekennzeichnet?
A Frauen sind häufiger betroffen.
B Das Durchschnittsalter der Patienten liegt zwischen 25 und 35 Jahren.
C Eine ZSCR bei jüngeren Patienten tritt nur einseitig auf.
D Schwangerschaft ist kein Risikofaktor für die Bildung einer ZSCR.
E Nichtbehandelte arterielle Hypertonie ist ein Risikofaktor.

2

Einer der folgenden Befunde

zählt nicht zu den typischen

klinischen Symptomen bei

zentraler seröser Chorioretinopathie

(ZSCR). Welcher?
A Zentralskotom
B Metamorphopsien
C Hypermetropisierung
D Farbsinnstörung
E Photopsien

3

Eine zentrale seröse Chorioretinopathie (ZSCR) präsentiert sich ...
A ... akut oder chronisch, aber immer beidseitig.
B ... typischerweise bei der akuten Form mit Abhebung der neurosensorischen Netzhaut und gutem Visus.
C ... nie mit Pigmentepithelabhebungen.
D ... bei der chronischen Form immer mit kleinen subretinalen Blutungen.
E ... zu einem höheren Prozentsatz mit einer choroidalen Neovaskularisation.

\section{4}

Welche Aussage zur Pathogenese und Diagnose der zentralen serösen Chorioretinopathie (ZSCR) trifft zu?
A Die primäre Pathologie wird ausschließlich im retinalen Pigmentepithel vermutet.

B Die Fluoresceinangiografie muss bei der akuten Form extrafoveoläre RPE-Leckagepunkte zeigen.

C Die Autofluoreszenzuntersuchung ist zur Unterscheidung einer akuten von einer chronischen Form hilfreich.

D Das mfERG spielt bei der Diagnosestellung eine wichtige Rolle.

E Die Perimetrie hat einen großen Stellenwert bei der Diagnosestellung.

5

Welche Aussage zur Behandlung der zentralen serösen Chorioretinopathie (ZSCR) trifft zu?
A Bei der akuten Form bildet sich nur ein geringer Teil spontan zurück.

B Die exogene Zufuhr von Kortikosteroiden soll vermieden und Stress abgebaut werden.

C Die fokale Lasertherapie spielt keine Rolle bei der Behandlung.

D Die photodynamische Therapie (PDT) eignet sich nur für die akute Form.

E Anti-VEGF-Medikamente werden nie mit der PDT kombiniert. 


\section{CME-Fragen}

\section{6}

Eine der folgenden Aussagen zur Grubenpapille ist nicht korrekt. Welche?
A Die Grubenpapille ist meist einseitig.

B Die Grubenpapille entsteht durch Fehlbildung von anatomischen Strukturen an der Papille.

C Die Grubenpapille kann sich als seröse Makulaabhebung präsentieren.

D Nach Diagnosestellung muss die Grubenpapille sofort behandelt werden.

E Grubenpapillen können mit einer juxtapapillären temporalen Laserkoagulation behandelt werden.
7

Wodurch ist die myope Makulopathie gekennzeichnet?
A Die pathologische Myopie betrifft $50 \%$ der Kurzsichtigen, von denen 5 bis $10 \%$ eine myope choroidale Neovaskularisation entwickeln.

B Verdünnung der Aderhaut und geringere Dichte des RPE begünstigen die Bildung einer myopen Makulopathie nicht.

C Die Fluoresceinangiografie ist die wichtigste Untersuchung zur Diagnosestellung.

D Der Durchmesser der subretinalen Membranen beträgt meist $500 \mu \mathrm{m}$.

E Die SD-OCT-Untersuchung ist die wichtigste Untersuchung zur Diagnosestellung.

8

Welche Aussage zur Therapie der myopen Makulopathie trifft zu?
A Die Laserkoagulation kommt bei juxta- und subfoveolären Läsionen zum Einsatz.

B Die photodynamische Therapie ist eine zugelassene Therapie bei myoper CNV.

C Anti-VEGF-Medikamente sind nicht zugelassen.

D Es gibt keine wirksame Therapie.

E Das SD-OCT ist zur Verlaufskontrolle ungeeignet.

9

Nur eine der folgenden Aussage zu Aderhautfalten trifft zu. Welche?
A Orbitale Tumoren führen immer zu Aderhautfalten.
B Aderhautfalten treten nur unilateral auf.
C Aderhautfalten können gemeinsam mit einem Papillenödem auftreten.
D Aderhautfalten treten immer gemeinsam mit subretinalen Blutungen auf.
E Die typische Anordnung der Aderhautfalten ist längs.

\section{0}

Wodurch sind Angioid Streaks gekennzeichnet?
A Sie entstehen aufgrund von Degenerationen von kollagenen Fasern in der Bruch-Membran.

B Es zeigen sich meist bilateral bräunliche, radiär von der Papille ausgehende Streifen.

C Sie treten nicht bei Diabetes mellitus auf.

D Es treten immer Symptome auf.

E Angioid Streaks sind immer mit Systemerkrankungen vergesellschaftet. 